\title{
Absence of Mutations of the BRAF Gene and Constitutive Activation of Extracellular-Regulated Kinase in Malignant Melanomas of the Uvea
}

\author{
Anette Weber, Ulrich R. Hengge, Doris Urbanik, Annett Markwart, \\ Alireza Mirmohammadsaegh, Martin B. Reichel, Christian Wittekind, \\ Peter Wiedemann, and Andrea Tannapfel
}

Department of Otorhinolaryngology, Head and Neck Surgery (AW), Institute of Pathology (DU, AMa, CW, AT), and Department of Ophthalmology (PW), University of Leipzig, Leipzig, and Department of Dermatology (URH, AMi), Heinrich-Heine-University, Düsseldorf, and Department of Ophthalmology (MBR), Clinic of Konstanz, Konstanz, Germany

\begin{abstract}
SUMMARY: The v-raf murine sarcoma viral homolog B1 (BRAF) gene, one of the human isoforms of RAF, is activated by Ras, leading to cooperative effects in cells responsive to growth factor signals. Recently, somatic missense mutations of the $B R A F$ gene have been detected in more than $66 \%$ of malignant melanomas of the skin. We analyzed 42 malignant melanomas of the uvea, 3 corresponding liver metastases, and 10 cutaneous melanomas for possible BRAF mutations: after microdissection, mutation analysis of BRAF and KRAS was performed. The expression of extracellular-regulated kinase 1 and 2 (ERK1/2), an important downstream point of convergence in the Ras-RAF-MEK-Erk pathway, was analyzed immunohistochemically. Interestingly, we failed to detect activating BRAF mutations in uvea melanomas and their corresponding liver metastases. There were no mutations of $B R A F$ in corresponding non-neoplastic uvea specimens, although we detected three $B R A F$ mutations in sporadic cutaneous melanoma that led to a substitution of valine by glutamic acid at position 599 (V599E). KRAS mutations were detected in 1 of 10 cutaneous melanoma but not in uveal or metastatic melanoma. Despite the lack of activating mutations in the $B R A F$ gene, we identified constitutively activated ERK in almost all (86\%) uveal melanoma tissues tested but not in corresponding normal retina or uveal cells. Our data indicate that $B R A F$ gene mutations are rare to absent events in uveal melanoma. The finding of activated Erk suggests a causative role for MAPK activation in uveal melanoma independent of activating BRAF or RAS mutations. (Lab Invest 2003, 83:1771-1776).
\end{abstract}

$M$ alignant melanoma of the uvea is an uncommon tumor with a prevalence of 6 in 1,000,000 per year, a frequency of approximately $12 \%$ that of cutaneous melanomas (Singh and Topham, 2003a). There is a great variation in its incidence around the world, being lowest in Africa and highest in Scandinavian countries, reflecting the racial differences in pigmentation. The role of UV light in the pathogenesis of uveal melanomas is still under intense discussion. Uveal melanomas, arising from the ciliary body and choroid, are highly aggressive tumors, causing death in up to $50 \%$ of the patients as a result of metastatic disease (Singh and Topham, 2003b). The clinical features of uveal melanomas located in the posterior part of the eye (visual loss, visual field deficits) may lead to early recognition and, therefore, effective local treat-

\section{DOI: 10.1097/01.LAB.0000101732.89463.29}

Received September 15, 2003.

$A W$ and URH contributed equally to the study. This work was supported by the Bundesministerium für Bildung und Forschung (BMBF), Interdisciplinary Centre for Clinical Research (IZKF) at the University of Leipzig (01KS9504/1, Project D01).

Address reprint requests to: Dr. A. Tannapfel, Institute of Pathology, University of Leipzig, Liebigstr. 26, D-04103 Leipzig, Germany. E-mail: tana@medizin.uni-leipzig.de ment of the primary tumor. However, the disease tends to spread, most often to the liver (Hendrix et al, 1998).

The differences between cutaneous and uveal melanomas are intriguing (McCartney, 1995). Both share a common cell of origin but differ substantially in their behavior and response to chemotherapy. There is increasing evidence that this is related to differences in their molecular phenotype. For example, p53 mutations seem to be less common in uveal melanomas than in skin melanomas, probably because of the lack of UV exposure in the uvea (Chowers et al, 2002). Additional differences exist in proliferation-associated proteins such as c-myc and cyclin D1 (Cree, 2000; White et al, 1998). Overexpression of the former molecule is associated with a poor prognosis in skin melanomas but is associated with a good prognosis in uveal melanomas, although there is considerable genetic heterogeneity within each type. Epidemiologically, the two entities also behave differently. The prevalence of cutaneous melanoma has increased dramatically during the last several decades, whereas such a trend is less evident with ocular melanomas (Singh and Topham, 2003a). In contrast, the incidence of cutaneous melanomas seems to be dependent on latitude, presumably reflecting exposure to ultraviolet 
light, as opposed to uveal melanomas (Desmond and Soong, 2003).

The Ras/Raf/extracellular-regulated kinase (ERK) pathway is relevant for proliferation, survival, angiogenesis, and oncogenesis and seems to be critically involved in the development of melanoma (Easty and Bennett, 2001). Although mutations of the $v$-raf murine sarcoma viral homolog $B 1$ (BRAF) have recently been described in approximately $66 \%$ of malignant cutaneous melanomas and $82 \%$ of melanocytic nevi (Davies et al, 2002), activating Ras and BRAF were also found in benign nevi (Pollock et al, 2003). However, recent data from melanoma-prone families suggests that BRAF is unlikely to be a melanoma susceptibility gene (Laud et al, 2003). Instead, BRAF mutations may be viewed as a progression marker, because they are only weakly expressed in the radial (initial) growth phase of cutaneous melanomas and become highly prevalent in the vertical (advanced) growth stages (Dong et al, 2003; Satyamoorthy et al, 2003). Our study was performed to assess BRAF and KRAS mutations and to analyze downstream signaling of the Ras/Raf/ERK pathway in uveal melanomas.

\section{Results}

\section{BRAF and KRAS Gene Alterations}

Genomic DNA from uveal melanoma and corresponding normal tissue was analyzed for BRAF gene mutations. We failed to detect somatic BRAF mutations in all 42 analyzed patients. In liver metastases of uveal melanomas, BRAF was of wild type as well. BRAF mutations were found in $3(30 \%)$ of 10 sporadic cutaneous melanomas. As expected, mutations represented the $T 1796 \mathrm{~A}$ mutation, resulting in the substitution of valine 599 by glutamate, a previously documented hotspot (sequence version NM004333.1). In all three cases, the normal epithelium of the same patient exhibited wild-type BRAF.

We did not detect KRAS mutations in uveal melanomas and corresponding metastases. In one sporadic cutaneous melanoma, a mutation of codon 12 (GGT $\rightarrow$ GTT) was detected, leading to a glycine to valine amino acid substitution. This particular cutaneous melanoma had wild-type BRAF. In summary, there was no significant correlation between the mutation status of BRAF, tumor stage or grade, or other histopathologic factors (tumor size, vascular invasion, multiplicity, desmoplastic reaction).

\section{Expression of Active ERK}

Given the importance of the Ras-Raf pathway for proliferation, survival, angiogenesis, and oncogenesis, we analyzed downstream signaling (which has been described to occur via activating BRAF mutations) in more detail. Active (ie, phosphorylated) ERK (p42/44 MAPK) was observed in 36 (86\%) of 42 uveal melanomas in tumors of different cellular types with a nearly homogeneous intratumoral expression (Fig. 2, $\mathrm{G}, \mathrm{H}$, and J), whereas unphosphorylated ERK showed baseline levels (Fig. 2, E, F, and I). The active protein was observed in the epithelioid, spindle, and mixed cell type. Melanomas of the choroidea or ciliary body were also positive.

In cutaneous melanomas and corresponding metastases, active phosphorylated ERK immunostaining was observed as well (data not shown), whereas, as expected, unphosphorylated ERK was observed in normal melanocytes of the ciliary body and the iris (Fig. 2, A and B). In contrast to the strong immunostaining of phosphorylated ERK in uveal melanomas, active ERK was almost negative in normal pigmented uveal or retinal cells (Fig. 2, C and D).

\section{Discussion}

Although BRAF was examined in a variety of human malignancies (Brose et al, 2002; Davies et al, 2002; Yuen et al, 2002) and found to be mutated in approximately $66 \%$ of cutaneous malignant melanomas, our study confirms the lack of BRAF mutations in uveal melanoma (Cohen et al, 2003; Edmunds et al, 2003) and elaborates on the constitutive activation of the Ras-RAF-MEK-Erk pathway by demonstrating phosphorylated Erk. In contrast to cutaneous melanoma or colon cancer (Yuen et al, 2002), we failed to detect $B R A F$ mutation in this tumor entity as has been recently reported (Cohen et al, 2003; Edmunds et al, 2003). Cutaneous melanoma specimens had a comparable frequency of mutated BRAF when compared with the literature (Brose et al, 2002; Davies et al, 2002). Our BRAF mutation of skin melanomas occurred at nucleotide 1796, leading to a $T$ to $A$ change in exon 15 of the BRAF gene. It has been previously shown that this mutation in the activation segment is a hotspot for BRAF mutation in human cancer, leading to a conversion of valine 599 to glutamic acid (Davies et al, 2002; Yuen et al, 2002).

Albeit uveal and cutaneous melanomas share a common cell of origin, they differ substantially in their behavior and response to chemotherapy. Our data support the idea that this is related to differences in their molecular phenotype. Besides BRAF, several other genetic differences exist between skin and uveal melanomas (Chowers et al, 2002; Cree, 2000). There is no association between uveal melanomas and the p16-related mutations found in cutaneous melanomas. p53 or pRB mutations are rare events in uveal as compared with skin melanomas (Chowers et al, 2002; Cree, 2000). Chromosomal imbalances, monosomy 3, trisomy 8 , and structural or numerical abnormalities of chromosome 6 have been reported for uveal but not for skin melanomas (Metzelaar-Blok et al, 2002). In addition, very recently, BRAF has been ruled out as a melanoma susceptibility gene (Lang et al, 2003; Laud et al, 2003).

The high frequency of $B R A F$ activation mutations in cutaneous melanomas and also melanocytic nevi provide new evidence that the Ras-RAF-MEK-Erk pathway plays a critical role in tumorigenesis of melanomas (Rajagopalan et al, 2002). In uveal melanomas, activating mutations in Ras are rarely observed. As a consequence of its mutation, BRAF gains the ability to 
Anti-ERK
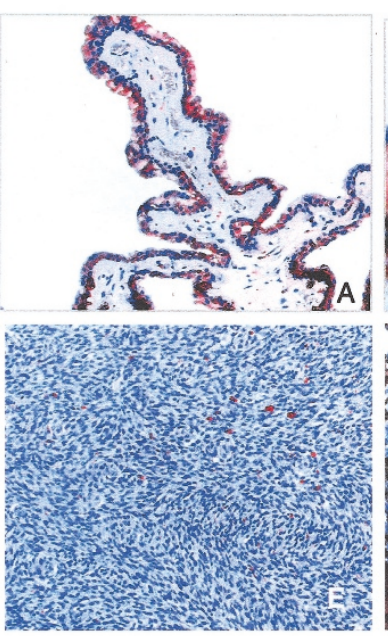
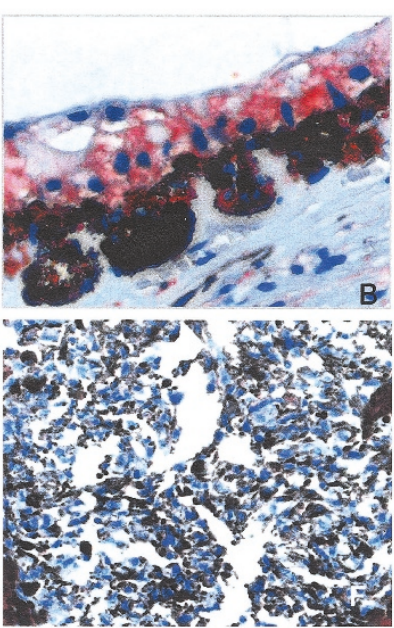

.
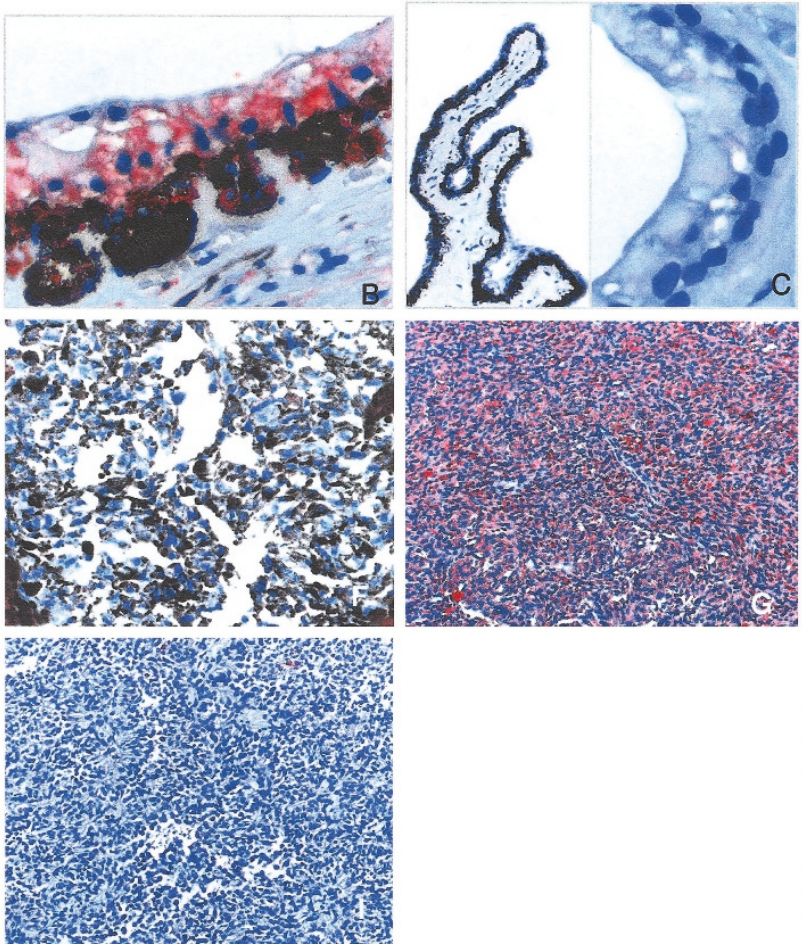

Anti-phospho ERK
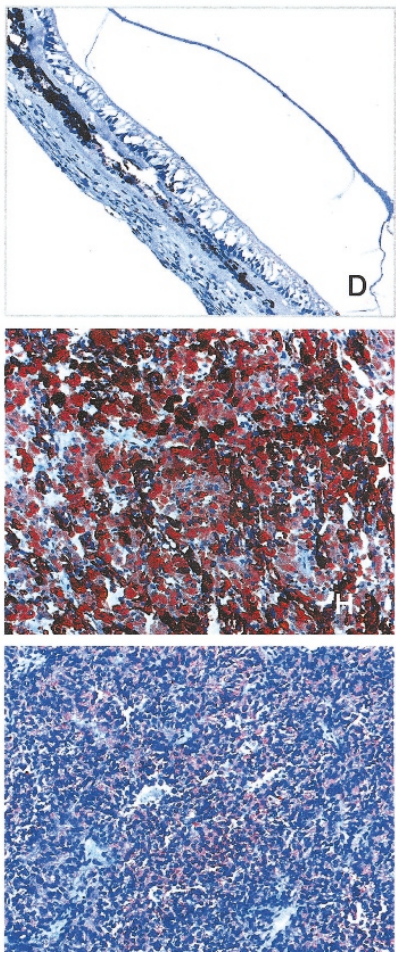

Figure 1.

Immunostaining of the anti-p44/42 MAPK (extracellular-regulated kinase; ERK) and anti-phospho-p44/42 MAPK MAPK (active ERK). A and B, Perinuclear and slight nuclear ERK staining (red reaction product) of the pigmented cells of the ciliary body (A) and the iris (B); original magnifications: $A, \times 2.5$; B, $\times 40$ ). C and D, Weak to absent active ERK staining of the pigmented cells of the ciliary body (C) and the normal choroidea (D); original magnifications: $\mathrm{C}, \times 2.5 ;$ inset, $\times 63$; D: $\times 10$ ). E, $\mathrm{F}$, and I, Weak ERK staining in uveal melanomas of the spindle cell type $(\mathrm{E})$, epithelioid type with pigmentation $(\mathrm{F})$, and mixed (fascicular) tumor type (I); original magnifications: E, $\times 20 ; F, \times 20 ; I, \times 20) . G, H$, and J, Same tumors as in E, F, and I. Strong active ERK staining in uveal melanomas of the spindle cell type (G), epithelioid type with pigmentation $(\mathrm{H})$, and mixed tumor type $(\mathrm{J})$; original magnifications: $\mathrm{G}, \times 20 ; \mathrm{H}, \times 20 ; \mathrm{J}, \times 20$ ).

interact with MEK and several other signaling molecules without the prerequisite of being phosphorylated. In the present study we show that most uveal melanomas signal through constitutively activated Erk1/2 (p44/42 MAPK). One may speculate that for uveal melanomas, neither RAS nor BRAF mutations may be a prerequisite for the activation of Ras-RAFMEK-Erk pathway. Alternative ways of activation of ERK have recently been detected. The inhibition of Rac1 or Cdc42 signaling led to MAPK and Erk activation via a pathway involving $\mathrm{PI}(3) \mathrm{K}$, Akt, Raf, and MEK (Peyssonnaux and Eychene, 2001; Rul et al, 2002). Interestingly, Cdc42 was implicated in the activation of p38, which in relation to the activation status of Erk, determined whether a tumor cell was actively proliferating (high Erk/p38 ratio) or was dormant (low Erk/p38 ratio) (Aguirre-Ghiso et al, 2003). These challenging assumptions are further substantiated by the description of Raf mutants that dissociate MEK/ERK activation from malignant transformation but not from proliferation (Dhillon et al, 2003).

In conclusion, we have demonstrated that in contrast to cutaneous melanomas, BRAF gene mutations are rare to absent events in uveal melanomas. However, the Erk1/2 pathway downstream of Ras is constitutively active in both melanoma entities. Identification and understanding of the precise cellular events that precede these two independent mechanisms may provide clues to the inhibition of Ras/Raf/ERK signaling using small molecule receptor tyrosine kinase inhibitors or siRNA.

\section{Materials and Methods}

\section{Patients and Tissue Samples}

Tissue samples (cross sections) of 42 patients with primary malignant melanoma of the uvea were collected between 1990 and 2002. Tumor typing and staging were performed using Union Internationale Contre le Cancer [International Union Against Cancer (UICC) (2002)] criteria. The majority of the primary tumors were located in the choroidea $(n=26)$, followed by the ciliary body $(n=12)$ and iris $(n=4)$. According to UICC (2002), the staging was as follows: 15 patients presented with a Stage I tumor, 21 with Stage II, and 6 with Stage III disease. In three patients, corresponding liver metastases were available for analysis. We further analyzed 10 randomly selected patients with sporadic cutaneous melanoma from the files of the Institute of Pathology. All patients were informed of special examination of tumor samples, which was in accordance with the ethical standards of the Committee on Human Experimentation of the University of Leipzig. 


\section{DNA Samples}

For each sample, microdissection of formalin-fixed paraffin-embedded tissue was performed as described previously (Tannapfel et al, 2003) using a UV laser microbeam (PALM, Bernried, Germany) (Fig. 1, A to $C)$. Using the UV laser beam at a high repetition rate (approximately 20 pulses/second), a circle was cut around the target cells (Fig. 1, B and C). This resulted in complete separation of the target population from neighboring tissue (Fig. 1, B and C).

\section{Mutation Analysis}

All pre-PCR tissue was handled in an environment free of PCR products. All samples were coded, and the investigator was blinded to all patients' clinical details. Deparaffinized microdissected tissue was recovered during a 15-minute incubation with xylene followed by centrifugation for 5 minutes at $14,000 \mathrm{rpm}$ two times. The tissue pellet was then washed twice in absolute ethanol fol- lowed by two washes in PBS. The pellet was incubated with 10 pellet volumes (approximately $500 \mu \mathrm{l}$ ) of lysis buffer [0.32 м sucrose, $10 \mathrm{~mm}$ Tris- $\mathrm{HCl}, 1 \%(\mathrm{v} / \mathrm{v})$ Triton $\mathrm{X}-100$ ] and 0.2 volumes of proteinase $\mathrm{K}$ (final concentration $400 \mu \mathrm{g} / \mathrm{ml}$ ) for 2 to 3 days at $37^{\circ} \mathrm{C}$. DNA was phenol-chloroform extracted and precipitated in ethanol using conventional techniques. The resulting DNA pellet was resuspended in $50 \mu \mathrm{l}$ of TE buffer, $\mathrm{pH} 7.4(10 \mathrm{~mm}$ Tris- $\mathrm{HCl}, \mathrm{pH} 7.4,1 \mathrm{~mm}$ EDTA, pH 8.0). DNA samples were stored at $-20^{\circ} \mathrm{C}$. Using matched samples (tumor and normal tissue from the same patient) we screened for exon 11 and exon 15 BRAF mutations. PCR primers were designed to amplify the exon plus at least $50 \mathrm{bp}$ of flanking intronic sequence according to previously published protocols (Davies et al, 2002). The primers were adopted from those published in the literature to omit analyzing the BRAF pseudogene (Davies et al, 2002; Naoki et al, 2002; Yuen et al, 2002). The following primers were used-exon 15: forward: GCCCCTCGATAACCAATIT, reverse: TCATCCATATTTCACAT-

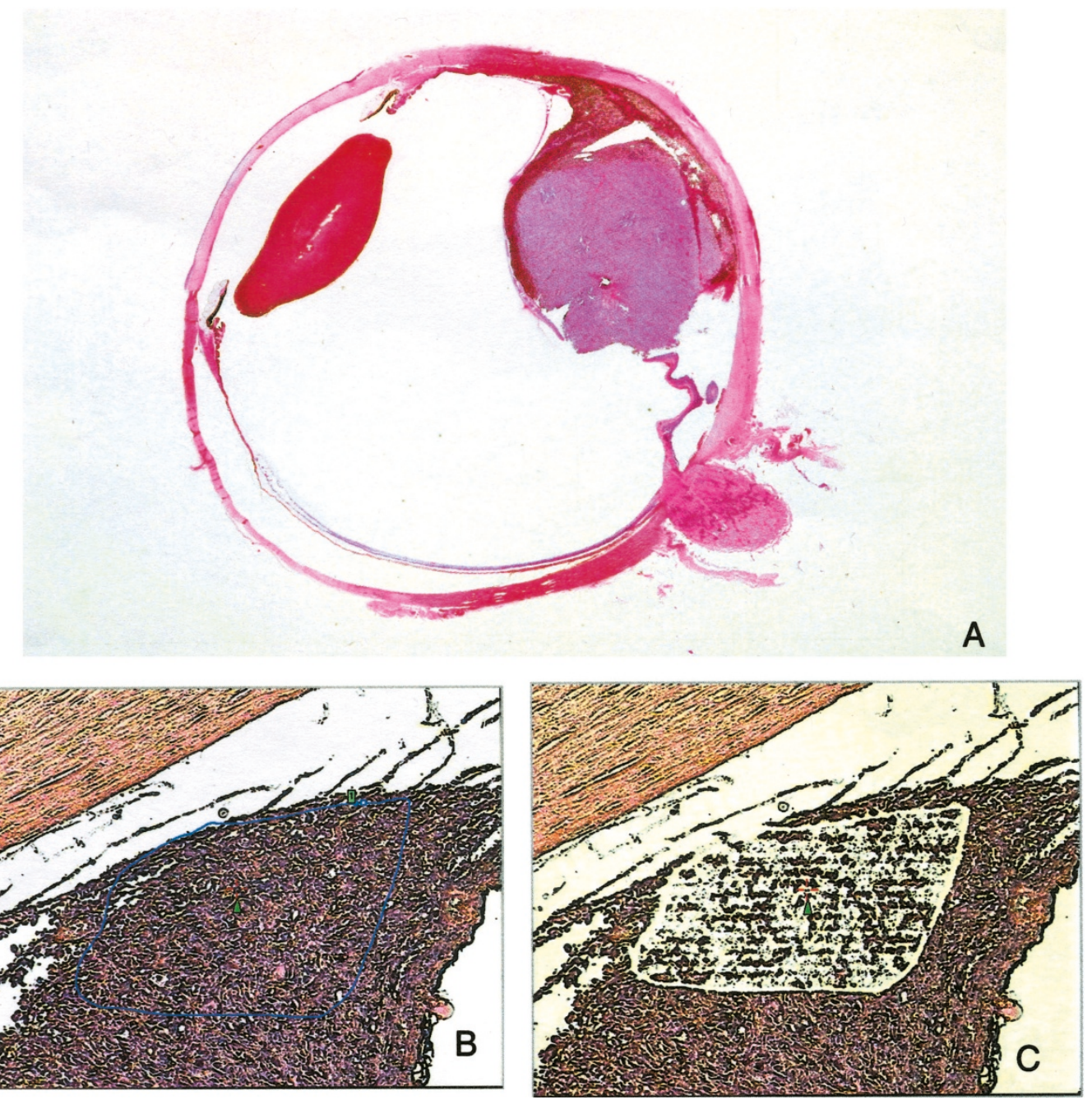

Figure 2.

A, Horizontal section of an eye (pupil-optic nerve section) with a malignant melanoma of the choroidea. The tumor was $0.6 \mathrm{~cm}$ in diameter without extraocular extension (pT1a, according to UICC 2002). Hematoxylin and eosin: original magnification, $\times 1$. B and C, Microdissection of the tumor compartments. Microdissection of the melanoma cells. The outlined areas (B) were microdissected (C) by the laser system (PalmMicrobeam System) and catapulted as described in "Materials and Methods." 
TCCCTA; exon 11: forward: TCCCTCTCAGGCATAAGGTAA, reverse: CGAACAGTGAA TATTTCCTITGAT. A total of $20 \mathrm{ng}$ of genomic DNA from all test samples was amplified using standard PCR conditions. Sequencing analysis was performed as described previously (Tannapfel at al, 2003).

For KRAS, the first exon was amplified by PCR using primers designed to avoid amplification of the KRAS pseudogene. The primers used were 5'-ATTATAAGGCCTGCTGAAAATG-ACTGA-3' (upstream primer) and 5'-ATATGCATATTAAAACAAGATTTACCT-CTA-3' (downstream primer), giving a 155-bp product. Amplification was performed using a touchdown PCR technique (McCartney, 1995; Metzelaar-Blok et al, 2002) from $63^{\circ} \mathrm{C}$ to $53^{\circ} \mathrm{C}$ for 10 cycles, followed by 30 cycles at $94^{\circ} \mathrm{C}, 53^{\circ} \mathrm{C}$, and $72^{\circ} \mathrm{C}$. PCR products were purified using the Qiaquick PCR purification kit (Qiagen, Hilden, Germany) and sequenced as described previously (Tannapfel et al, 2003; Weber et al, 2003).

As a positive control for ras mutation analysis, DNA from colon carcinoma cell lines SW480 (Clontech, Palo Alto, California) and HCT116 (American Type Culture Collection, Rockville, Maryland) with known KRAS mutations at codon 12 (GTT) and codon 13 (GAC) respectively, were used. Negative controls, without DNA, were run as controls for contamination.

\section{Immunohistochemistry}

For immunohistochemistry, paraffin-embedded melanoma sections were deparaffinized in xylolisopropanol solution. To expose cryptic antigens, the sections were cooked in citrate buffer $(0.1 \mathrm{M}$ citric acid, $0.1 \mathrm{~m}$ sodium nitrate) for 10 minutes. Blocking was performed with $10 \%$ normal goat serum. The first antibody dilution (p44/42 MAPK, Phospho-p44/42 MAPK; Cell Signaling, Boston, Massachusetts) was 1:200 in 1\% BSA. Anti-phospho-p44/42 MAPK specifically recognizes the dually phosphorylated, active form of MAPK (also known as p44/ERK1 and p42/ ERK2) enzymes. Incubation was performed for 1 hour at room temperature. The secondary antibody (Dako, Hamburg, Germany) was diluted 1:40 and incubated for 1 hour at room temperature. For signal augmentation, the APAAP complex (Dako) was diluted 1:80 and incubated for 30 minutes at room temperature. As chromogen, new fuchsin development solution was used, and counterstaining was performed using methylene blue solution. Sections known to stain positively were included in each batch, and negative controls were also performed by replacing the primary antibody with mouse or goat ascites fluid (Sigma-Aldrich Biochemicals, St. Louis, Missouri).

\section{References}

Aguirre-Ghiso JA, Estrada Y, Liu D, and Ossowski L (2003). ERK(MAPK) activity as a determinant of tumor growth and dormancy: Regulation by p38(SAPK). Cancer Res 63:16841695.

Brose MS, Volpe P, Feldman M, Kumar M, Rishi I, Gerrero R, Einhorn E, Herlyn M, Minna J, Nicholson A, Roth JA, Albelda
SM, Davies H, Cox C, Brignell G, Stephens P, Futreal PA, Wooster R, Stratton MR, and Weber BL (2002). BRAF and RAS mutations in human lung cancer and melanoma. Cancer Res 62:6997-7000.

Chowers I, Folberg R, Livni N, and Pe'er J (2002). p53 Immunoreactivity, Ki-67 expression, and microcirculation patterns in melanoma of the iris, ciliary body, and choroid. Curr Eye Res 24:105-108.

Cohen Y, Goldenberg-Cohen N, Parrella P, Chowers I, Merbs SL, Pe'er J, and Sidransky D (2003). Lack of BRAF mutation in primary uveal melanoma. Invest Ophthalmol Vis Sci 44: 2876-28768.

Cree IA (2000). Cell cycle and melanoma: Two different tumours from the same cell type. J Pathol 191:112-114.

Davies H, Bignell GR, Cox C, Stephens P, Edkins S, Clegg S, Teague J, Woffendin H, Garnett MJ, Bottomley W, Davis N, Dicks E, Ewing R, Floyd Y, Gray K, Hall S, Hawes R, Hughes J, Kosmidou V, Menzies A, Mould C, Parker A, Stevens C, Watt S, Hooper S, Wilson R, Jayatilake H, Gusterson BA, Cooper C, Shipley J, Hargrave D, Pritchard-Jones K, Maitland N, Chenevix-Trench G, Riggins GJ, Bigner DD, Palmieri G, Cossu A, Flanagan A, Nicholson A, Ho JW, Leung SY, Yuen ST, Weber BL, Seigler HF, Darrow TL, Paterson H, Marais R, Marshall CJ, Wooster R, Stratton MR, and Futreal PA (2002). Mutations of the BRAF gene in human cancer. Nature 417:949-954.

Desmond RA and Soong SJ (2003). Epidemiology of malignant melanoma. Surg Clin North Am 83:1-29.

Dhillon AS, Meikle S, Peyssonnaux C, Grindlay J, Kaiser C, Steen $H$, Shaw PE, Mischak $H$, Eychene A, and Kolch $W$ (2003). A Raf-1 mutant that dissociates MEK/extracellular signal-regulated kinase activation from malignant transformation and differentiation but not proliferation. Mol Cell Biol 23:1983-1893.

Dong J, Phelps RG, Qiao R, Yao S, Benard O, Ronai Z, and Aaronson SA (2003). BRAF oncogenic mutations correlate with progression rather than initiation of human melanoma. Cancer Res 63:3883-3885.

Easty DJ and Bennett DC (2001). Protein tyrosine kinases in malignant melanoma. Melanoma Res 10:401-411.

Edmunds SC, Cree IA, Di Nicolantonio F, Hungerford JL, Hurren JS, and Kelsell DP (2003). Absence of BRAF gene mutations in uveal melanomas in contrast to cutaneous melanomas. Br J Cancer 88:1403-1405.

Hendrix MJ, Seftor EA, Seftor RE, Gardner LM, Boldt HC, Meyer M, Pe'er J, and Folberg R (1998). Biologic determinants of uveal melanoma metastatic phenotype: Role of intermediate filaments as predictive markers. Lab Invest 78:153-163.

Lang J, Boxer M, and MacKie R (2003). Absence of exon 15 BRAF germline mutations in familial melanoma. Hum Mutat 21:327-330.

Laud K, Kannengiesser C, Avril MF, Chompret A, StoppaLyonnet D, Desjardins L, Eychene A, Demenais F, Lenoir GM, and Bressac-De Paillerets B (2003). BRAF as a melanoma susceptibility candidate gene? Cancer Res 63:3061-3065.

McCartney AC (1995). Pathology of ocular melanomas. Br Med Bull 51:678-693.

Metzelaar-Blok JA, Jager MJ, Moghaddam PH, van der Slik AR, and Giphart MJ (2002). Frequent loss of heterozygosity 
on chromosome $6 p$ in uveal melanoma. Hum Immunol 60: 962-969.

Naoki K, Chen TH, Richards WG, Sugarbaker DJ, and Meyerson M (2002). Missense mutations of the BRAF gene in human lung adenocarcinoma. Cancer Res 62:7001-7003.

Peyssonnaux C and Eychene A (2001). The Raf/MEK/ERK pathway: New concepts of activation. Biol Cell 93:53-62.

Pollock PM, Harper UL, Hansen KS, Yudt LM, Stark M, Robbins CM, Moses TY, Hostetter G, Wagner U, Kakareka J, Salem G, Pohida T, Heenan P, Duray P, Kallioniemi O, Hayward NK, Trent JM, and Meltzer PS (2003). High frequency of BRAF mutations in nevi. Nat Genet 33:19-20.

Rajagopalan H, Bardelli A, Lengauer C, Kinzler KW, Vogelstein B, and Velculescu VE (2002). Tumorigenesis: RAF/ RAS oncogenes and mismatch-repair status. Nature 418: 934.

Rul W, Zugasti O, Roux P, Peyssonnaux C, Eychene A, Franke TF, Lenormand P, Fort P, and Hibner U (2002). Activation of ERK, controlled by Rac1 and Cdc42 via Akt, is required for anoikis. Ann NY Acad Sci 973:145-148.

Satyamoorthy K, Li G, Gerrero MR, Brose MS, Volpe P, Weber BL, Van Belle P, Elder DE, and Herlyn M (2003). Constitutive mitogen-activated protein kinase activation in melanoma is mediated by both BRAF mutations and autocrine growth factor stimulation. Cancer Res 63:756-759.
Singh AD and Topham A (2003a). Incidence of uveal melanoma in the United States: 1973-1997. Ophthalmology 110: 956-961.

Singh AD and Topham A (2003b). Survival rates with uveal melanoma in the United States: 1973-1997. Ophthalmology 110:962-965.

Tannapfel A, Sommerer F, Benicke M, Katalinic A, Uhlmann D, Witzigmann H, Hauss J, and Wittekind C (2003). Mutations of the BRAF gene in cholangiocarcinoma but not in hepatocellular carcinoma. Gut 52:706-712.

Weber A, Langhanki L, Sommerer F, Markwarth A, Wittekind C, and Tannapfel A (2003). Mutations of the BRAF gene in squamous cell carcinoma of the head and neck. Oncogene 22:4757-4759.

White VA, McNeil BK, and Horsman DE (1998). Acquired homozygosity (isodisomy) of chromosome 3 in uveal melanoma. Cancer Genet Cytogenet 102:40-45.

Yuen ST, Davies H, Chan TL, Ho JW, Bignell GR, Cox C, Stephens P, Edkins S, Tsui WW, Chan AS, Futreal PA, Stratton MR, Wooster R, and Leung SY (2002). Similarity of the phenotypic patterns associated with BRAF and KRAS mutations in colorectal neoplasia. Cancer Res 62:64516455 . 